\title{
On the SUSY Accompanied Neutrino Exchange Mechanism of Neutrinoless Double Beta Decay
}

\author{
M. Hirsch, H.V. Klapdor-Kleingrothaus and S.G. Kovalenko* \\ Max-Planck-Institut für Kernphysik, P.O. 1039 80, D-69029, Heidelberg, \\ Germany \\ ${ }^{*}$ Joint Institute for Nuclear Research, Dubna, Russia
}

\begin{abstract}
The neutrinoless double beta decay $(0 \nu \beta \beta)$ induced by light Majorana neutrino exchange between decaying nucleons, accompanied by the squark exchange inside one nucleon, recently discussed by Babu and Mohapatra, is carefully analyzed both from the particle and nuclear physics sides. New nuclear matrix elements relevant to this mechanism are calculated. We extend the analysis to include mixing of light neutrinos with heavy and "sterile" neutrinos. It introduces another supersymmetric (SUSY) contribution to $0 \nu \beta \beta$. We discuss constraints on the $\not R_{p}$ MSSM parameters imposed by the current experimental limit on $0 \nu \beta \beta$ decay half-life of ${ }^{76} \mathrm{Ge}$.
\end{abstract}

Neutrinoless double beta $(0 \nu \beta \beta)$ decay is a sensitive probe of physics beyond the standard model, since it violates lepton number. Recent experimental progress has pushed the existing half-life limits of $0 \nu \beta \beta$ decay beyond $T_{1 / 2}(0 \nu \beta \beta) \geq 7.4 \times 10^{24}$ years and further progress can be expected in the near future [1]. This experimental result casts stringent constraints on new physics. Particularly, for the conventional mechanism of $0 \nu \beta \beta$-decay with massive Majorana neutrino exchange between decaying nucleons (see fig. 1) it implies an upper bound on the neutrino mass below $1 \mathrm{eV}$ [1]. (There exist, however, other mechanisms which might induce $0 \nu \beta \beta$ decays as well [2], [3].)

In this paper we study contributions to $0 \nu \beta \beta$ decay within the R-parity violating Minimal Supersymmetric Standard Model ( $\left.\not R_{p} \mathrm{MSSM}\right)$. The $\not R_{p} \mathrm{MSSM}$ has been extensively discussed in the literature since it has very interesting phenomenological [4] and cosmological [5] implications. It also gives a very natural framework for rare lepton number violating processes and particularly $0 \nu \beta \beta$ decay [6]-10].

The supersymmetric mechanism of $0 \nu \beta \beta$ decay was first proposed by Mohapatra [6] and later studied in more details in Refs. [7], 8]. In Ref. [9] it was shown that the gluino exchange contribution to $0 \nu \beta \beta$-decay leads to a very 
stringent limit on the first generation $\not R_{p}$ Yukawa coupling $\lambda_{111}^{\prime} \leq 3.9 \cdot 10^{-4}$. Recently, Babu and Mohapatra [10] found another contribution comparable in size with the gluino exchange. It allows one to set stringent limits on combinations of the intergeneration $\not R_{p}$-Yukawa couplings such as $\lambda_{11 i}^{\prime} \lambda_{1 i 1}^{\prime}$, where $i$ denotes generations.

However, in ref. [10] limits were deduced using a simplified estimation of the nuclear structure matrix elements. The approach used in [10] is based on a simple replacement of the virtual particle momenta and energies by the (estimated) Fermi momentum $p_{F}$ and energy $E_{F}$ of a nucleon inside the nucleus, therefore neglecting essentially all nuclear structure effects. Since the stringent constraints found in [10] may have important consequences for the $R_{p}$ MSSM phenomenology, it is desirable to substantiate these results by detailed calculations.

In this letter we present the results of detailed particle and nuclear physics calculations relevant to the contribution of the diagrams in fig. 2 to $0 \nu \beta \beta$ decay. We extend the previous work to include mixing of the standard light neutrinos with exotic heavy neutrinos, neutralinos or some other neutral heavy particles or light "sterile" neutrino singlets [11. Inclusion of mixing leads to the new slepton exchange diagram in fig. (2.b).

The $R_{p}$ MSSM is an extension of the MSSM which results from the inclusion of explicit $R$-parity violating $\left(\not R_{p}\right)$ terms $W_{\mathbb{R}_{p}}$ into the superpotential $W=$ $W_{M S S M}+W_{R_{p}}$, where

$$
W_{\not R_{p}}=\lambda_{i j k} L_{i} L_{j} \bar{E}_{k}+\lambda_{i j k}^{\prime} L_{i} Q_{j} \bar{D}_{k}+\lambda_{i j k}^{\prime \prime} \bar{U}_{i} \bar{D}_{j} \bar{D}_{k}
$$

Indices $i, j, k$ stand for generations. $L, Q$ denote lepton and quark doublet superfields and $\bar{E}, \bar{U}, \bar{D}$ lepton and up, down quark singlet superfields. The first two terms in eq. (I) lead to lepton number violation, while the last one violates baryon number. For $0 \nu \beta \beta$ decay only the $\lambda$ and $\lambda^{\prime}$ type couplings are of relevance.

Mixing between scalar superpartners $\tilde{f}_{L, R}$ of the left and right-handed fermions $f_{L, R}$ will play a crucial role in our subsequent consideration. It occurs due to non-diagonality of the mass matrix which can be written as

$$
\mathcal{M}_{\tilde{f}}^{2}=\left(\begin{array}{cc}
m_{\tilde{f}_{L}}^{2}+m_{f}^{2}-0.42 D_{Z} & -m_{f}\left(A_{f}+\mu \tan \beta\right) \\
-m_{f}\left(A_{f}+\mu \tan \beta\right) & m_{\tilde{f}_{R}}^{2}+m_{f}^{2}-0.08 D_{Z}
\end{array}\right) .
$$

Here, $f=d, s, b, e, \mu, \tau$ and $\tilde{f}$ are their superpartners. $D_{Z}=M_{Z}^{2} \cos 2 \beta$ with $\tan \beta=\left\langle H_{2}^{0}\right\rangle /\left\langle H_{1}^{0}\right\rangle$ being the ratio of vacuum expectation values of the two 
Higgs doublets, $m_{\tilde{f}_{L, R}}$ are soft sfermion masses, $A_{f}$ are soft SUSY breaking parameters describing the strength of trilinear scalar interactions, and $\mu$ is the supersymmetric Higgs(ino) mass parameter. Once sfermion mixing is included, the current eigenstates $\tilde{f}_{L}, \tilde{f}_{R}$ become superpositions of the mass eigenstates $\tilde{f}_{i}$ with the masses $m_{\tilde{f}_{i}}$ and the corresponding mixing angle $\theta^{f}$ is defined as

$$
\begin{array}{r}
m_{\tilde{q}_{1,2}}^{2}=\frac{1}{2}\left[m_{L L}^{2}+m_{R R}^{2} \mp \sqrt{\left(m_{L L}^{2}-m_{R R}^{2}\right)^{2}+4 m_{L R}^{4}}\right] \\
\sin 2 \theta^{f}=\frac{2 m_{(f) L R}^{2}}{m_{\tilde{f}_{1}}^{2}-m_{\tilde{f}_{2}}^{2}},
\end{array}
$$

where $m_{L R}^{2}, m_{L L}^{2}, m_{R R}^{2}$ denote the $(1,2),(1,1),(2,2)$ entries of the mass matrix ((2)) .

Now it is straightforward to find the effective 4 -fermion $\nu-u-d-e$ vertex induced by the sfermion exchange in the diagrams presented in fig. 2. The corresponding effective Lagrangian, after a Fiertz rearrangement, takes the form

$$
\begin{aligned}
\mathcal{L}_{S U S Y}^{e f f}(x) & =\frac{G_{F}}{\sqrt{2}}\left[\frac{1}{4}\left(\eta_{(q) L R}^{n j}-4 \eta_{(l) L R}^{n j}\right) \cdot U_{n i}^{*} \cdot\left(\bar{\nu}_{i}\left(1+\gamma_{5}\right) e_{j}^{c}\right)\left(\bar{u}\left(1+\gamma_{5}\right) d\right)-\right. \\
& -2 \eta_{(l) L L}^{n j} \cdot U_{n i} \cdot\left(\bar{\nu}_{i}\left(1-\gamma_{5}\right) e_{j}^{c}\right)\left(\bar{u}\left(1+\gamma_{5}\right) d\right)+ \\
& +\frac{1}{2} \eta_{(q) R R}^{n j} \cdot U_{n i}\left(\bar{\nu}_{i} \gamma^{\mu}\left(1+\gamma_{5}\right) e_{j}^{c}\right)\left(\bar{u} \gamma_{\mu}\left(1-\gamma_{5}\right) d\right)+ \\
& \left.+\frac{1}{8} \eta_{(q) L R}^{n j} \cdot U_{n i}^{*} \cdot\left(\bar{\nu}_{i} \sigma^{\mu \nu}\left(1+\gamma_{5}\right) e_{j}^{c}\right)\left(\bar{u} \sigma_{\mu \nu}\left(1+\gamma_{5}\right) d\right)\right]
\end{aligned}
$$

The $\not R_{p}$ MSSM parameters $\eta$ and neutrino mixing matrix $U_{i j}$ are defined as follows

$$
\begin{aligned}
\eta_{(q) L R}^{n j} & =\sum_{k} \frac{\lambda_{j 1 k}^{\prime} \lambda_{n k 1}^{\prime}}{2 \sqrt{2} G_{F}} \sin 2 \theta_{(k)}^{d}\left(\frac{1}{m_{\tilde{d}_{1}(k)}^{2}}-\frac{1}{m_{\tilde{d}_{2}(k)}^{2}}\right), \\
\eta_{(q) R R}^{n j} & =\sum_{k} \frac{\lambda_{j 1 k}^{\prime} \lambda_{n 1 k}^{\prime}}{2 \sqrt{2} G_{F}}\left(\frac{\sin \theta_{(k)}^{d}}{m_{\tilde{d}_{1}(k)}^{2}}+\frac{\cos \theta_{(k)}^{d}}{m_{\tilde{d}_{2}(k)}^{2}}\right) \\
\eta_{(l) L R}^{n j} & =\sum_{k} \frac{\lambda_{k 11}^{\prime} \lambda_{n j k}}{2 \sqrt{2} G_{F}} \sin 2 \theta_{(k)}^{e}\left(\frac{1}{m_{\tilde{e}_{1}(k)}^{2}}-\frac{1}{m_{\tilde{e}_{2}(k)}^{2}}\right), \\
\eta_{(l) L L}^{n j} & =\sum_{k} \frac{\lambda_{k 11}^{\prime} \lambda_{n k j}}{2 \sqrt{2} G_{F}}\left(\frac{\cos \theta_{(k)}^{e}}{m_{\tilde{e}_{1}(k)}^{2}}+\frac{\sin \theta_{(k)}^{e}}{m_{\tilde{e}_{2}(k)}^{2}}\right)
\end{aligned}
$$




$$
\nu_{i}^{0}=\sum_{j} U_{i j} \nu_{j}
$$

Here $\eta_{(f) L R}$ denotes the contribution vanishing in the absence of $\tilde{f}_{L}-\tilde{f}_{R}-$ mixing while $\eta_{(f) L L}$ and $\eta_{(f) R R}$ in this limit correspond to the $\tilde{f}_{L}$ and $\tilde{f}_{R}$ exchange contribution in fig. 2. We use the notations $d_{(k)}=d, s, b$ and $e_{(k)}=e, \mu, \tau$. Due to the antisymmetry of the Yukawa coupling $\lambda_{n j k}$ in $n j$ it follows that $\eta_{(l) L R}^{n n}=0$. This is an essential difference between the slepton $\tilde{l}_{L}-\tilde{l}_{R}$ and the squark $\tilde{q}_{L}-\tilde{q}_{R}$ contributions. The latter is not imposed to vanish at any combination of indexes.

The matrix element of the SUSY accompanied neutrino exchange mechanism can then be calculated according to the the diagrams in fig. 2 with the point-like 4-fermion vertex described by the effective Lagrangian eq. (14) with the sfermion exchange parts in the top. The bottom parts of these diagrams is the standard model charged current (SMCC) interaction. Applying the standard procedure (for details see [9]), one can get the matrix element $\mathcal{R}_{0 \nu \beta \beta}\left(0^{+} \rightarrow 0^{+}\right)$of the $0 \nu \beta \beta$ decay for $0^{+} \rightarrow 0^{+}$transitions. For two outgoing electrons in $\mathrm{S}$-wave states it takes the form

$$
\begin{aligned}
\mathcal{R}_{0 \nu \beta \beta}\left(0^{+} \rightarrow 0^{+}\right) & =C_{0 \nu} f_{A}^{2}\left[a \cdot \bar{e}\left(1+\gamma_{5}\right) e^{c}+b \cdot \bar{e} \gamma_{0} \gamma_{5} e^{c}\right] \\
a & =\left(4 \eta_{(l) L R}^{n 1}-\eta_{(q) L R}^{n 1}\right) U_{n i}^{*} U_{e i} \mathcal{M}_{1}^{(i)}\left(m_{e} R\right)^{-1}+ \\
& +\left(\eta_{(q) R R}^{n 1}-\delta_{n e}\right) U_{n i} U_{e i} \frac{m_{\nu_{i}}}{m_{e}} \mathcal{M}_{2}^{(i)} \\
b & =4 \eta_{(l) L L}^{n 1} U_{n i} U_{e i} \frac{m_{\nu_{i}}}{m_{e}} \mathcal{M}_{3}^{(i)}
\end{aligned}
$$

The term proportional to $\delta_{n e}$ corresponds to the ordinary neutrino exchange contribution with two standard model charged current vertices in fig. 1. The normalization factor $C_{0 \nu}$ is defined as $C_{0 \nu}=\left(G_{F}^{2} 2 m_{e}\right) /(8 \sqrt{2} \pi R)$. We would like to stress that the terms proportional to $b$ in eq. (10) can, in principle, be discriminated from the terms proportional to $a$ (particularly from the ordinary mass mechanism of $0 \nu \beta \beta$-decay) by measuring the angular correlation between the two outgoing electrons.

The following nuclear matrix elements are involved in the calculation of $R_{0 \nu \beta \beta}$ in eq. (10)

$$
\begin{aligned}
\mathcal{M}_{1}^{(i)} & =\alpha_{1}\left[\mathcal{M}_{T^{\prime}}^{(i)}+\frac{1}{3} \mathcal{M}_{G T^{\prime}}^{(i)}\right] \\
\mathcal{M}_{2}^{(i)} & =\alpha_{2} \mathcal{M}_{F}^{(i)}-\mathcal{M}_{G T}^{(i)}, \quad \mathcal{M}_{3}^{(i)}=\alpha_{3} \mathcal{M}_{F}^{(i)}
\end{aligned}
$$


They are defined by (summation over nucleons is suppressed)

$$
\begin{aligned}
\mathcal{M}_{F}^{(i)} & =<0_{f}^{+}\left\|h_{+}\left(r_{a b}, m_{\nu_{i}}\right) \tau_{a}^{+} \tau_{b}^{+}\right\| 0_{i}^{+}> \\
\mathcal{M}_{G T}^{(i)} & =<0_{f}^{+}\left\|h_{+}\left(r_{a b}, m_{\nu_{i}}\right)\left(\vec{\sigma}_{a} \vec{\sigma}_{b}\right) \tau_{a}^{+} \tau_{b}^{+}\right\| 0_{i}^{+}> \\
\mathcal{M}_{G T^{\prime}}^{(i)} & =<0_{f}^{+}\left\|h_{R}\left(r_{a b}, m_{\nu_{i}}\right)\left(\vec{\sigma}_{a} \vec{\sigma}_{b}\right) \tau_{a}^{+} \tau_{b}^{+}\right\| 0_{i}^{+}> \\
\mathcal{M}_{T^{\prime}}^{(i)} & =<0_{f}^{+}\left\|h_{T^{\prime}}\left(r_{a b}, m_{\nu_{i}}\right)\left[\left(\vec{\sigma}_{a} \hat{\mathbf{r}}_{a b}\right)\left(\vec{\sigma}_{b} \hat{\mathbf{r}}_{a b}\right)-\frac{1}{3}\left(\vec{\sigma}_{a} \vec{\sigma}_{b}\right)\right] \tau_{a}^{+} \tau_{b}^{+}\right\| 0_{i}^{+}>.
\end{aligned}
$$

Neutrino potentials can be written in the integral form

$$
\begin{aligned}
h_{+}\left(r_{a b}, m_{\nu_{i}}\right) & =\frac{2}{\pi} R \int_{0}^{\infty} d q \cdot q^{2} \frac{j_{0}\left(q r_{a b}\right) f^{2}\left(q^{2}\right)}{\omega(\omega+\bar{A})}, \\
h_{R}\left(r_{a b}, m_{\nu_{i}}\right) & =\frac{2}{\pi} \frac{R^{2}}{m_{P}} \int_{0}^{\infty} d q \cdot q^{4} \frac{j_{0}\left(q r_{a b}\right) f^{2}\left(q^{2}\right)}{\omega(\omega+\bar{A})}, \\
h_{T^{\prime}}\left(r_{a b}, m_{\nu_{i}}\right) & =\frac{2}{\pi} \frac{R^{2}}{m_{P}} \int_{0}^{\infty} d q \cdot q^{4} \frac{j_{0}\left(q r_{a b}\right)-3 j_{1}\left(q r_{a b}\right)}{\omega(\omega+\bar{A})} f^{2}\left(q^{2}\right) .
\end{aligned}
$$

Here, $\omega=\sqrt{q^{2}+m_{\nu_{i}}^{2}} ; j_{k}(q r)$ are spherical Bessel functions and $R_{0}$ is the nuclear radius, introduced to make the matrix elements dimensionless. The following notations are used: $\mathbf{r}_{a b}=\left(\vec{r}_{a}-\vec{r}_{b}\right), r_{a b}=\left|r_{a b}\right|, \hat{\mathbf{r}}_{a b}=\mathbf{r}_{a b} / r_{a b}$. The above formulae have been written in the closure approximation which is well motivated for the $0 \nu \beta \beta$ decay [2]. $\bar{A}$ in eq. (19) is the average intermediate state energy.

Note that the nuclear matrix element $\mathcal{M}_{T^{\prime}}^{(i)}$ has never been considered in the literature before. We have calculated the nuclear matrix elements relevant for our subsequent numerical analysis using the pn-QRPA model of [13]. We take into account both short-range correlations and finite nucleon size effects. The latter is described by introducing nucleon form factors in momentum space. In the present case these are the SMCC form factors $F_{(V, A)}^{C C}\left(q^{2}\right)$ and the isovector scalar and pseudoscalar current form factors $F_{P, S}^{3}\left(q^{2}\right)$. For all form factors we take, as usual, a dipole parameterization: $F_{i}^{a}\left(q^{2}\right) / F_{i}^{a}(0)=f\left(q^{2}\right)=$ $\left(1+q^{2} / m_{A}^{2}\right)^{-2}$ with $m_{A}=0.85 \mathrm{GeV}$. The q-dependent factor $f\left(q^{2}\right)$ is included in the definition of the neutrino potentials in eqs. (19). The form factor normalizations are $F_{V(A)}^{C C}(0)=f_{V(A)} \approx 1(1.261) . F_{S, P}^{3}(0)$ can be calculated within the conventional non-relativistic quark model or the bag model. We take their numerical values from ref. [14] $F_{S}^{3}(0) \approx 0.48 F_{P}^{3}(0) \approx 4.41$. These values correspond to the bag model calculations. 
The nucleon structure coefficients in (13) are defined as $\alpha_{1}=\left(F_{P}^{(3)} /\left(2 f_{A}\right)\right.$, $\alpha_{2}=\left(f_{V} / f_{A}\right)^{2}, \alpha_{3}=\left(f_{V} / f_{A}\right)\left(F_{S}^{(3)} / f_{A}\right)$.

Now having the $0 \nu \beta \beta$ matrix element completely specified the inverse half life $T_{1 / 2}(0 \nu \beta \beta)$ can be written as

$$
T_{1 / 2}^{-1}(0 \nu \beta \beta)=|a|^{2} G_{01}+|b|^{2} h_{9} G_{09}+\operatorname{Re}\left(a^{*} b\right) h_{6} G_{06},
$$

where $h_{6}=m_{e} R / 8, h_{9}=\left(m_{e} R\right)^{2} / 16$, and phase space factors $G_{0 i}$ are given in ref. [2].

The formulae presented above describe the contribution of the diagrams in fig. 2 for a general neutrino content (see eq. (9)). We now turn to a particular case and assume that all neutrino mass eigenstates fall into two groups: light neutrinos $m_{\nu_{i}}<10 \mathrm{MeV}$ and heavy neutrinos $m_{\nu_{i}}>10 \mathrm{GeV}$. We denote them as $\nu_{i}$ and $N_{i}$, respectively. There might be also a "sterile" neutrino $\nu_{s}$ with respect to the SM gauge group. Assume further, that the light neutrinos have non-negligible mixing with some heavy neutrinos $N_{i}$ or probably with the "sterile" neutrino $\nu_{s}$. Than the neutrino composition (9) and the unitarity relation for the mixing matrix $U_{i j}$ can be written as

$$
\begin{aligned}
\nu_{i}^{0} & =\sum_{j}^{\prime} U_{i j} \nu_{j}+\sum_{j}^{\prime \prime} U_{i j} \nu_{j}+U_{i s} \nu_{s}, \\
\sum_{j} U_{i j}^{*} U_{e i} & =\sum_{j}^{\prime} U_{i j}^{*} U_{e i}+\Delta_{i}, \quad \Delta_{i}=\sum_{j}^{\prime \prime} U_{i j}^{*} U_{e i}+U_{i s} \nu_{s} .
\end{aligned}
$$

Such a structure of the neutrino sector breaks contributions of each nuclear matrix elements in eq. (10) into two pieces with a simple dependence on the neutrino mass. As an example of this effect consider the first term in eq. (11)

$$
\sum_{i} U_{n i}^{*} U_{e i} \mathcal{M}_{1}^{(i)}=\sum_{i}^{\prime} U_{n i}^{*} U_{e i} \mathcal{M}_{1}^{\nu}+\sum_{i}^{\prime \prime} \frac{U_{n i}^{*} U_{e i}}{M_{N_{i}}^{2}} \mathcal{M}_{1}^{N} .
$$

The "sterile" neutrino does not contribute being a SM singlet. The matrix elements are $\mathcal{M}_{1}^{\nu}=\mathcal{M}_{1}^{(i)}\left(m_{\nu}=0\right)$ and $\mathcal{M}_{1}^{N}=\lim _{M_{N}^{2} \rightarrow \infty} M_{N} \mathcal{M}_{1}^{(i)}\left(M_{N}\right)$. They do not depend on neutrino masses. Proceeding in a similar way with the other matrix elements in eq. (10) and substituting the result in the half-life formula eq. (22) we obtain a polynomial in $m_{\nu}$ and $M_{N}^{-1}$. Assume light neutrinos $\nu$ to be very light while heavy neutrinos $N$ to be very heavy so that one can neglect terms depending on these masses. Than, keeping only leading terms we retain

$$
T_{1 / 2}^{-1}(0 \nu \beta \beta)=G_{01} \mathcal{M}_{1}^{\nu}\left(m_{e} R\right)^{-1}\left(4 \bar{\eta}_{(l)}-\bar{\eta}_{(q)}+\eta_{(q)}\right)^{2},
$$


where eq. (25) and the property $\eta_{(l) L R}^{n n}=0$ have been used. We denoted $\eta_{(q)}=\eta_{(q) L R}^{11}$ and introduced the effective parameters as

$$
\bar{\eta}_{(l, q)}=\sum_{n} \Delta_{n} \eta_{(l, q) L R}^{n 1}
$$

For the $\bar{\eta}_{(l)}$ summation starts from $n=2$. The nuclear matrix element $\mathcal{M}_{1}^{\nu}$ in eq. (26) can be directly obtained from eq. (13) for $\mathcal{M}_{1}^{(i)}$ as explained after eq. (25). This matrix element has never been calculated in the literature before. Its value calculated in the pn-QRPA for the particular case of ${ }^{76} \mathrm{Ge}$ analyzed below is $\mathcal{M}_{1}^{(\nu)}\left({ }^{76} \mathrm{Ge}\right)=2.1$. ए

Now we are ready to discuss constraints on the parameters in eq. (26) imposed by the current experimental lower half-life limit. We use the result from the Heidelberg-Moscow ${ }^{76} \mathrm{Ge}$ experiment $T_{1 / 2}^{0 \nu \beta \beta}\left({ }^{76} \mathrm{Ge}, 0^{+} \rightarrow 0^{+}\right)>7.4 \times$ $10^{24}$ years $90 \%$ c.l.

Disregarding a situation with unnatural fine-tuning between the different terms in eq. (26) one can extract individual limits, numerically $\eta_{(q)} \leq$ $2.9 \times 10^{-8}, \bar{\eta}_{(l)} \leq 7.2 \times 10^{-9}$. These constraints lead, in principle, to a multidimensional exclusion curve. A simplified picture can be obtained under some reasonable assumptions. Assume all the MSSM mass parameters in eqs. (5) and (2), (3) to be approximately equal to the "effective" SUSY breaking scale $\Lambda_{S U S Y}$. Than we get a simplified set of constraints

$$
\lambda_{11 i}^{\prime} \lambda_{1 i 1}^{\prime} \leq \epsilon_{i}^{\prime}\left(\frac{\Lambda_{S U S Y}}{100 G e V}\right)^{3}, \quad \Delta_{n} \lambda_{311}^{\prime} \lambda_{n 13} \leq \epsilon\left(\frac{\Lambda_{S U S Y}}{100 G e V}\right)^{3} .
$$

In the last equation we kept only the term corresponding to $\tilde{\tau}$ exchange. For the known values of SM quark and lepton masses in the following we use $m_{d_{1}}=m_{d}=7.5 \mathrm{MeV}, m_{d_{2}}=m_{s}=150 \mathrm{MeV}, m_{d_{3}}=m_{b}=4.5 \mathrm{GeV}$ and $m_{\tau}=1.8 \mathrm{GeV}$. These masses are present in eqs. (22), (3). Then, $\epsilon_{1,2,3}^{\prime}=$ $\left\{6.4 \times 10^{-5}, 3.2 \times 10^{-6}, 1.1 \times 10^{-7}\right\}$ and $\epsilon=6.5 \times 10^{-8}$.

To obtain information on the $\not_{p}$ Yukawa couplings themselves one may use some reasonable values for $\Lambda_{S U S Y}$. If one takes, following ref. [10], the value $\Lambda_{S U S Y}=100 \mathrm{GeV}$ lying in the region of the current experimental lower bound for the SUSY particles, one gets $\lambda_{113}^{\prime} \lambda_{131}^{\prime} \leq 1.1 \times 10^{-7}, \lambda_{112}^{\prime} \lambda_{121}^{\prime} \leq 3.2 \times 10^{-6}$, $\lambda_{111}^{\prime 2} \leq 6.4 \times 10^{-5}$ and $\Delta_{n} \lambda^{\prime} \lambda \leq 6.5 \times 10^{-8}$. The former two limits correspond to those in ref. [10], but are somewhat weaker than quoted by Babu and Mohapatra. This difference can be partly traced back to our numerical value

\footnotetext{
${ }^{1}$ Recall that this numerical value corresponds to our dimensionless convention.
} 
of the matrix element, which turns out to be smaller than anticipated. The limit on $\lambda_{111}^{\prime 2}$ can be compared with the corresponding limit obtained from the gluino exchange diagram in ref. [9]. The latter being $\lambda_{111}^{\prime 2} \leq 1.52 \cdot 10^{-7}$ is much more stringent then the one derived here. More conservative estimations can be derived from eq. (28) implying $\Lambda_{S U S Y} \sim 1 \mathrm{TeV}$ motivated by the SUSY naturalness arguments. Then, $\lambda_{113}^{\prime} \lambda_{131}^{\prime} \leq 1.1 \times 10^{-4}, \lambda_{112}^{\prime} \lambda_{121}^{\prime} \leq 3.2 \times 10^{-3}$, $\lambda^{\prime 2}{ }_{111} \leq 6.4 \times 10^{-2}$ and $\Delta_{n} \lambda^{\prime} \lambda \leq 6.5 \times 10^{-4}$.

Neglecting mixing between the light SM non-singlet neutrinos and the nonstandard sector discussed above, one arrives at the case considered by Babu and Mohapatra [10]. In this case $\Delta_{n}=0$ and in turn $\bar{\eta}(l, q)=0$. Then the squark exchange diagram in fig. $2($ a) is the only contribution to eq. (26). Introducing mixing one can obtain new information about $\lambda$ type interactions. In this case, however, this information is accessible only in a form of some effective value of the $R_{p}$ Yukawa couplings $\Delta \lambda^{\prime} \lambda$. The upper limit for the couplings itself implies an extra uncertainty due to the unknown mixing factor $\Delta$.

The new contributions analysed in this letter together with the previously discussed in the literature [6]-9] complete the tree-level mechanisms of $0 \nu \beta \beta$ decay within the R-parity violating Minimal Supersymmetric Standard Model $\left(R_{p} \mathrm{MSSM}\right)$. A detailed presentation of the analysis will be given elsewhere.

In conclusion we would like to stress that the current experimental limit on $0 \nu \beta \beta$ decay half life allows one to establish rather stringent limits on $\not R_{p}$ violating SUSY interactions. This might be an additional valuable motivation for present and forthcoming $0 \nu \beta \beta$ decay experiments.

\section{ACKNOWLEDGMENTS}

We thank V.A. Bednyakov and R.N. Mohapatra for helpful discussions. The research described in this publication was made possible in part (S.G.K.) by Grant GNTP 215NUCLON from the Russian ministry of science. M.H. would like to thank the Deutsche Forschungsgemeinschaft for financial support by grants kl 253/8-1 and 446 JAP-113/101/0.

\section{References}

[1] HEIDELBERG-MOSCOW Collaboration: A. Balysh et al., Phys.Lett. B 356 (1995) 450;

H.V.Klapdor-Kleingrothaus, Progr.Part.Nucl.Phys. 32 (1994) p.261 and 
Proc. Workshop on Double Beta Decay and related topics, Trento, Italy, 1995, World Scientific, Singapore, in the press

[2] M. Doi, T. Kotani and E. Takasugi, Progr. Theor. Phys. Suppl. 83 (1985) 1.

[3] J. D. Vergados, Phys. Report, 133 (1986) 1; J.W.F. Valle, Prog. Part. Nucl. Phys. 26 (1991) 91; R.N. Mohapatra, Talk at the Workshop on Double Beta Decay and Related Topics, Trento, Italy, 1995 (to appear in the Workshop Proceedings).

[4] V. Barger, G.F. Guidice and T. Han, Phys. Rev. D 40 (1989) 2987; R. Barbieri and A. Masiero, Nucl.Phys. B267 (1986) 679; D.P. Roy, Phys. Lett. B 283 (1992) 270; F. Zwirner, Phys. Lett. B132 (1983) 103; H. Dreiner and P. Morawitz, Nucl. Phys. B 428 (1994) 31.

[5] A. Bouquet and P. Salati, Nucl.Phys. B284 (1987); A. Nelson and S.M. Barr, Phys.Lett. B258 (1991) 45; B.A. Campbell, S. Davidson, J. Ellis and K. Olive, Phys.Lett. B256 (1991) 457; CERN-preprint, CERN-TH6208-91; W. Fieschler, G. Guidice, R.G. Leigh and S. Paban, Phys.Lett. B258 (1991) 45.

[6] R.N. Mohapatra, Phys.Rev. D34 (1986) 3457.

[7] J.D Vergados, Phys.Lett. B184 (1987) 55.

[8] M. Hirsch, H.V. Klapdor-Kleingrothaus and S.G. Kovalenko, Phys.Lett. B352 (1995) 1.

[9] M. Hirsch, H.V. Klapdor-Kleingrothaus and S.G. Kovalenko, Phys. Rev. Lett., 75 (1995) 17; Preprint MPI-H-V 6-1995, Heidelberg, 1995, to appear in Phys. Rev. D (1995).

[10] K.S. Babu and R.N. Mohapatra, Phys.Rev.Lett., 75 (1995)2276;

[11] D. Caldwell and R.N. Mohapatra, Phys.Rev. D 48 (1993) 3259; J. Peltoniemi and J.W.F. Valle, Nucl.Phys. B 406 (1993) 409.

[12] L. Hall and M. Suzuki, Nucl.Phys. B 231 (1984) 419. D. Braham and L. Hall, Phys.Rev. D40 (1989) 2449. M. Bento, L.J. Hall and G.G. Ross, Nucl.Phys. B292 (1987) 400; G. Lazarides, P.K. Mohapatra, C. Panagiotakopoulos and Q. Shafi, Nucl.Phys. B323 (1989) 614. 
[13] K. Muto, E. Bender and H.V. Klapdor, Z. Phys. A 334 (1989) 177,187; M. Hirsch, K. Muto, T. Oda and H.V. Klapdor-Kleingrothaus, Z. Phys. A 347 (1994) 151

[14] S.L. Adler et al., Phys.Rev. D11 (1975) 3309.

\section{Figure Captions}

Fig.1 Feynman graphs for the conventional mechanism of $0 \nu \beta \beta$ decay by exchange of a massive Majorana neutrino.

Fig.2 Feynman graphs for the supersymmetry accompanied Majorana neutrino exchange mechanism of the $0 \nu \beta \beta$ decay corresponding to (a) squarks and (b) sleptons contributions. 
Figure 1

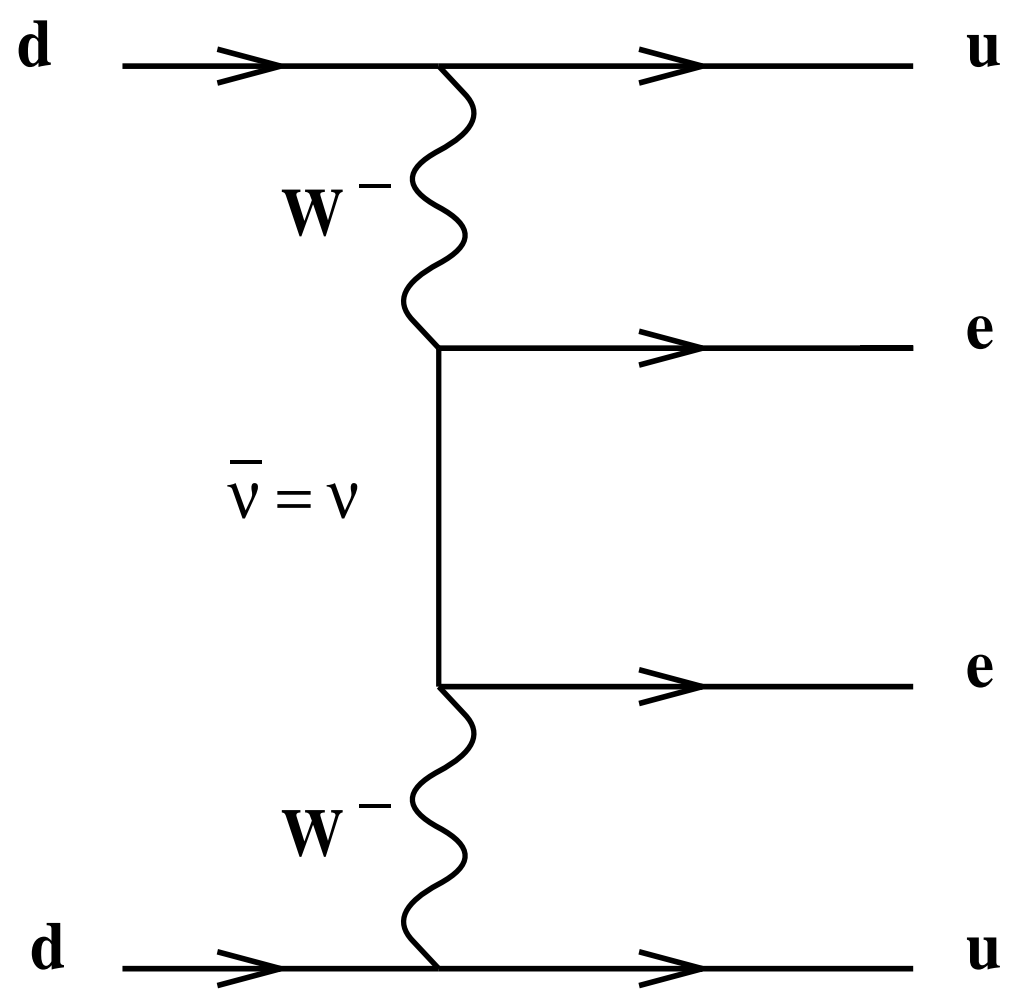


Figure 2.a

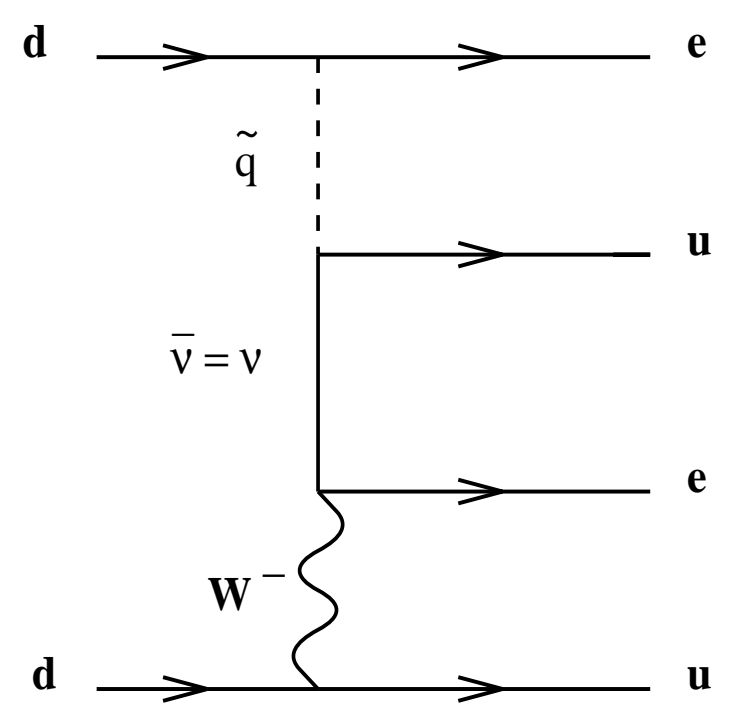

Figure 2.b

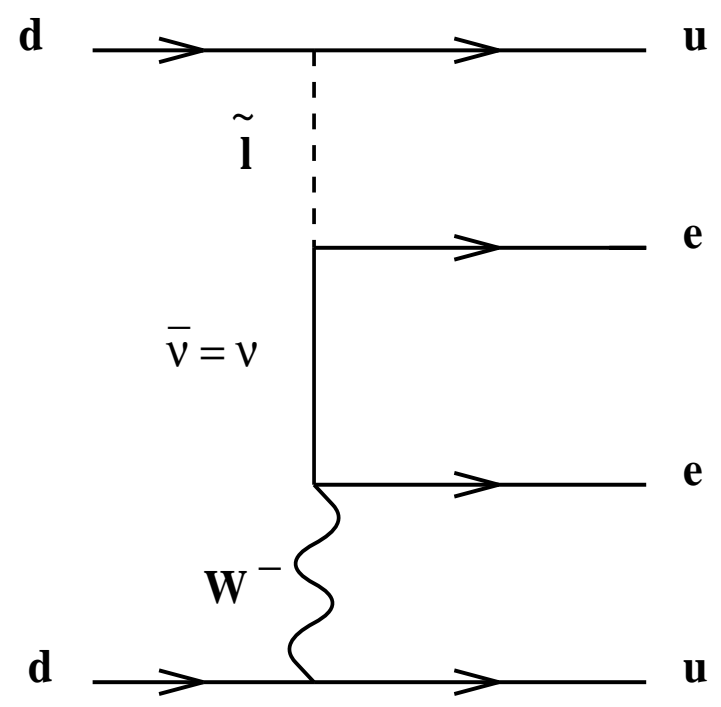

